\title{
La dynamique triangulaire dans le processus d'incorporation des demandeurs d'asile, les politiques migratoires et le rôle des organismes communautaires
}

\author{
Jacqueline Oxman-Martinez, Estibaliz Jimenez, Jill Hanley et Isabelle Bohard
}

\section{Résumé}

Cet article analyse comment le régime d'incorporation au Canada, à travers ses politiques et ses pratiques, facilite ou met à l'épreuve l'incorporation des demandeurs d'asile dès leur arrivée au Québec. Les auteurs examinent comment les intervenants des organisations communautaires perçoivent les obstacles dressés par le statut provisoire des demandeurs d'asile. Les dynamiques politiques enchevêtrées dans l'activité sociale où interagissent les demandeurs d'asile et les membres de la société d'accueil sont aussi analysées. L'étude se fonde sur les données et les résultats issus d'une revue documentaire et des entrevues faites auprès des intervenants et des représentants des différents paliers gouvernementaux.

\footnotetext{
Abstract

This article analyses how the present system for the integration of asylum seekers in Canada, as seen in policies and in actual practice, either facilitates or impedes the integration of asylum seekers right from the moment of their arrival in Quebec. The authors examine how advocates from community organizations perceive the obstacles inherent to the temporary status of asylum seekers. The article further analyses the political dynamics that are entangled in social activities where asylum seekers and members of the host community interact. The study is based on data and results from a literature review and interviews conducted with advocacy groups and representatives of the various levels of government.
}

$\mathrm{L}$ e présent article analyse le processus d'incorporation des demandeurs d'asile au Canada, dans la province 1 de Québec. Il est basé sur certains résultats de la recherche Canada's incorporation of asylum seekers: the role of the state versus civil society in the context of Québec (Oxman-Martinez, J., Lacroix, M., et Hanley, J. 2004-2008) financée par le Conseil de recherche en sciences humaines du Canada (CRSH). L'article décrit d'abord comment le régime d'incorporation du gouvernement canadien, par le biais de ses politiques et de ses pratiques, facilite ou entrave l'incorporation des demandeurs d'asile dès leur arrivée au Québec. Ensuite, il examine comment les organismes communautaires et non gouvernementaux répondent aux différents besoins de cette population en situation précaire, en particulier aux besoins des services sociaux et de santé, en assurant la transition d'un statut temporaire à un statut permanent. Enfin, il expose les lacunes rencontrées par les demandeurs d'asile telles que constatées par les intervenants clés des organisations communautaires.

En d'autres mots, cet article analyse l'incorporation des demandeurs d'asile qui font leur demande au moment de leur arrivée au Québec ${ }^{1}$. Ces personnes peuvent être des réfugiés au sens de la Convention relative au statut des réfugiés ${ }^{2}$, des personnes à protéger ${ }^{3}$, des cas comportant des considérations humanitaires ${ }^{4}$, des personnes issues de pays visés par un moratoire ${ }^{5}$ ou tout simplement des personnes cherchant à améliorer leurs conditions de vie.

Le gouvernement fédéral partage la responsabilité de l'immigration avec les provinces et les territoires ${ }^{6}$. La Constitution canadienne, en vertu de son article 95, fait de l'immigration un domaine de compétence partagée. Aux termes de ses obligations internationales, le gouvernement 
fédéral détermine qui est un réfugié au sens de la Convention relative au statut des réfugiés et quelles sont les personnes en situation semblable qui ont besoin de protection. D’ailleurs, le gouvernement fédéral est le seul responsable du traitement des demandes d'asile au Canada. Toutefois, l'Accord Canada-Québec relatif à l'immigration et à l'admission temporaire des aubains ${ }^{7}$ accorde au Québec la compétence exclusive des services d'intégration. De ce fait et en vertu de la Loi sur l'immigration au Québec ${ }^{8}$, le Québec a la responsabilité d'offrir des services d'accueil, d'intégration économique, linguistique et culturelle aux nouveaux arrivants qui s'établissent dans cette province afin de favoriser leur initiation à la vie québécoise. Toutefois, selon Lacroix "Au Québec, les services aux demandeurs d'asile ont systématiquement subi des coupures depuis l'Entente Canada-Québec de $1991^{9}$ ».

En outre, les demandeurs d'asile qui entament une procédure de revendication du statut de réfugié au Canada, procédure longue et incertaine, ne bénéficie pas de l'ensemble des services d'établissement et d'intégration économique et sociale de la part du gouvernement fédéral ni provincial. Par exemple, les demandeurs d'asile ne sont pas couverts par la Régie de l'assurance-maladie du Québec $(\mathrm{RAMQ})^{10}$, mais plutôt par le Programme fédéral de santé intérimaire (PFSI) géré par Citoyenneté et Immigration Canada (CIC). Le PFSI accorde aux demandeurs d'asile une couverture temporaire qui offre uniquement les soins de santé essentiels et urgents ${ }^{11}$ pour les individus qui n'ont pas de ressources financières. Cependant, l'accès des demandeurs d'asile aux soins de santé est, dans la pratique, limité par l'hésitation des services de santé à accepter les cartes PFSI, les barrières linguistiques pour communiquer leurs besoins, le manque de familiarité avec le système de santé, la peur de se voir refuser le statut de réfugié si l'on sollicite ces aides, le manque d'information et de connaissances de leurs droits et les barrières socio-culturelles ${ }^{12}$.

Les organismes communautaires offrent des services afin d'aider les demandeurs d'asile, mais sans soutien financier gouvernemental suffisant. Ceci nuit en partie au processus d'incorporation des demandeurs d'asile au Québec. Pourtant, le taux d'acceptation devant la Commission de l'immigration et du statut du réfugié (CISR) est de $47 \%{ }^{13}$, ce qui signifie que presque la moitié des personnes qui obtiendront ultérieurement la résidence permanente subira une carence des services d'assistance et d'intégration au début de leur processus d'incorporation dans la société québécoise.

Les demandeurs d'asile se heurtent au cadre politicolégal du Canada dans l'accès égalitaire aux services sociaux et de santé ainsi qu'à une information adéquate de leurs droits.

\section{Cadre conceptuel}

La majorité des travaux de recherche sur les migrants traduit une expérience complexe et douloureuse pour les demandeurs d'asile. Les exemples cités par Mestheneos et Ioannidi ${ }^{14}$ décrivent ces situations comme une période négative liée aux traumatismes antérieurs, à l'isolement produit par l'exclusion sociale et une nouvelle culture, une nouvelle langue, la discrimination provoquant un sentiment d'infériorité. Ce contexte de vulnérabilité est accentué par la fluidité du concept d'intégration. Cette notion varie selon les idéologies nationales et temporelles ${ }^{15}$. L'hétérogénéité attachée à cette définition dirige soit vers un processus d'assimilation et d'uniformisation soit vers une tolérance mutuelle qui préconise une diversité culturelle, une égalité des chances. La culture dominante joue un rôle majeur dans l'accès à la société d'accueil et relègue le demandeur à un état de dépendance et de subordination qui l'exclut de la société ${ }^{16}$. Le concept d'intégration implique un processus où les immigrants doivent s'adapter pour répondre aux valeurs et aux normes de la culture dominante, ce qui contribue à diluer leurs propres systèmes de valeurs et de pratiques pour les enregistrer dans un régime d'assimilation, voire d'acculturation. Au contraire, le processus d'incorporation encourage, dès la présentation de la demande d'asile, la participation active, l'égalité et le dialogue entre les cultures dans une société caractérisée par la diversité ${ }^{17}$.

Afin de considérer le demandeur d'asile comme un acteur à part entière, nous privilégierons l'emploi du concept d'incorporation ${ }^{18}$. Le bien-fondé de ce concept est donc relié au fait qu'il s'agit d'un processus qui permet de prendre en considération les dynamiques sociales et culturelles des différents acteurs, soit le demandeur d'asile, la société du pays d'accueil, les organismes communautaires qui viennent en aide aux demandeurs d'asile et les politiques d'immigration en vigueur. Cette perspective permet d'envisager le demandeur d'asile comme un participant actif dans son processus d'établissement susceptible de recevoir les mêmes privilèges et de jouer les mêmes rôles que l'ensemble de la population sans discriminations a priori.

Les observations permettent de constater que le délai d'attente, l'incertitude et la stratification dans l'accès aux divers services sociaux et de santé cantonnent les demandeurs d'asile dans un état de marginalisation qui intensifie le sentiment d'insécurité et d'exclusion ${ }^{19}$. Cette précarité affectera non seulement les relations dans l'alliance entre le demandeur et la société d'accueil, mais aussi, la capacité et les limites des organismes communautaires d'aider les demandeurs d'asile en raison de leurs restrictions structurelles $^{20}$ et le choix politico-économique des gouvernements. Lacroix précise que l'accès partiel aux services publics accentue les difficultés des demandeurs d'asile 
déjà fragilisés dans leur expérience post-migratoire et aggrave les effets des traumatismes pré-migratoires ${ }^{21}$. Cette précarité précédemment décrite déstabilise les rapports de pouvoir déjà affaiblis entre le demandeur, l'État et la société civile. Des obstacles et des lacunes entravent la participation civique de cette population dans le processus d'incorporation et accentuent la vulnérabilité des demandeurs d'asile provoquée par des restrictions majeures telles que les difficultés d'accès aux cours de francisation à temps plein, aux stages et aux services de recherche d'emplois, au logement social et au régime de santé québécois.

Le statut d'immigration juridique accordé aux individus influence leur capacité d'accéder aux services et ressources qui leur soit nécessaires pour participer intégralement à la société d'accueil et satisfaire leurs besoins fondamentaux. Le concept d'incorporation permet de prendre en compte l'interaction des individus et de la société d'accueil et d'examiner les rapports et les répercussions qui soutiennent la participation des migrants ou, au contraire, la briment. La légitimité pour les immigrants de contracter et de jouir des mêmes privilèges que l'ensemble des nationaux puise son efficacité dans l'élargissement des droits individuels reconnus dans la Charte canadienne des droits et libertés ${ }^{22}$. Les principes universels de droits humains établis par les instances internationales influencent davantage les politiques d'immigration nationales ${ }^{23}$. Contrairement à la Déclaration universelle des droits de l'homme, l'éventail d'options fournies par les services sociaux et de santé et les moyens pour y accéder au Canada varient selon le statut légal des individus, en allant de services d'urgence à d'autres plus complexes ${ }^{24}$. Cette légitimité prend place dans un contexte de monde globalisé où un concept plus universel issu des traités internationaux et discours transnationaux autorise ce déploiement de revendication et de légitimité 25 .

L'expansion des flux migratoires a bouleversé la définition étroite de la citoyenneté attachée aux limites historiques et territoriales des nationaux et modifié le modèle de citoyenneté classique. L'engagement des migrants, dans les différents champs de la société, et notamment dans les activités liées à la production économique, sociale et culturelle, implique une révision des représentations véhiculées par les politiques migratoires qui contredisent la réalité de l'implication sociale des migrants. L'observation des pratiques montre que ces dispositifs institutionnels génèrent un accès différencié aux services en dépit d'une présence marquée dans les activités sociales, économiques, éducatives et culturelles. Cette distinction renforce la disparité qui caractérise la vulnérabilité des personnes à statut d'immigration précaire, et ce, malgré les interactions constantes avec les membres de la société d'accueil.
L'élément-clé de ce cadre théorique - le concept d'incorporation - permet de mieux appréhender les interactions entre les individus et la société d'accueil tout en analysant les rapports et les impacts qui encouragent la participation des migrants ou au contraire, la contraignent.

\section{Méthodologie}

La méthodologie utilisée dans la recherche sur laquelle cet article est basé est exploratoire et qualitative. Elle puise dans les données de deux sources : une revue documentaire et des entrevues semi-structurées. La première comprend la recension des programmes et des services offerts au Québec autant par les différents gouvernements dans le cas de partage de compétences que par les organismes communautaires. Les entrevues sélectionnées aux fins de cet article, faites dans les deux langues officielles, ont pour objet de répondre aux deux questions de recherche suivantes :

- Quels sont les obstacles et les besoins des demandeurs d'asile dans le processus d'incorporation observés par les intervenants clés des organisations communautaires?

- Quel est le rôle des organisations communautaires dans le processus d'incorporation des demandeurs d'asile?

Les entrevues ont été faites d'abord auprès des informateurs-clés (cinq) qui travaillent dans des coalitions qui regroupent des organismes communautaires d'aide aux immigrants et réfugiés et ensuite auprès des intervenants qui travaillent directement avec les réfugiés. Un deuxième type d'entrevue a été adressé aux représentants des agences municipales, provinciales et fédérales (cinq) dans le but d'obtenir leur perspective concernant le régime d'incorporation, sa modification à travers le temps et les enjeux actuels. Nous avons rencontré un certain degré de saturation au niveau de l'information qui concordait avec nos recherches préalables ${ }^{26}$.

Les entrevues ont été enregistrées, transcrites puis codifiées en suivant la méthode classique de la théorie ancrée ${ }^{27}$. Une analyse de contenu d'abord verticale, ensuite horizontale, de thèmes émergents et récurrents a été faite à l'aide du logiciel Atlas.

\section{Identification des obstacles dans le processus d'incorporation des demandeurs d'asile}

Bien que l'histoire personnelle et l'expérience d'accueil puissent varier considérablement, chaque demandeur d'asile est confronté à plusieurs défis lors de son arrivée au Québec. Les intervenants communautaires consultés signalent, entre autres, la barrière de la langue, la difficulté de trouver un emploi, le manque d'information et de références, de même qu'une allocation familiale insuffisante. Plusieurs besoins fondamentaux des demandeurs ont été identifiés par les 
répondants, par exemple, l'aide financière, l'accès à la santé, au logement, aux cours de francisation, à la recherche d'emploi, à l'aide juridique et, finalement, à une représentation juridique adéquate. De plus, la difficulté d'accès aux services, le long délai de traitement de la demande du statut de réfugié et la séparation familiale se sont avérés des problèmes majeurs vécus par les demandeurs d'asile. Pour certains répondants, ces obstacles retardent le processus d'incorporation à la société québécoise.

\section{L'inadmissibilité aux programmes d'aide à l'intégration et à l'établissement}

Les demandeurs d'asile ne sont pas admissibles aux mêmes programmes gouvernementaux d'aide à l'intégration et à l'établissement offerts aux réfugiés parrainés par l'État ${ }^{28}$. Les répondants ont désigné cette exclusion relative des politiques comme étant un obstacle majeur à l'incorporation des demandeurs d'asile. Les services auxquels les demandeurs d'asile peuvent avoir accès sur le territoire québécois sont rares. Les informateurs-clés ont énuméré les suivants : le programme fédéral de santé intérimaire; certains services en sports, loisirs et bibliothèques offerts par les villes; les cours de français à temps partiel; l'hébergement temporaire; l'aide à la recherche d'un logement; les aides financière et juridique (bien qu'insuffisantes et inadéquates selon les répondants); ainsi que l'accès gratuit à l'école et aux différents programmes scolaires pour les jeunes de 6 à 16 ans sous la responsabilité des Commissions scolaires.

Les demandeurs d'asile ne bénéficient pas de la totalité des services d'intégration offerts aux nouveaux arrivants. Plusieurs informateurs-clés communautaires constatent dans leur pratique des coupures budgétaires du gouvernement du Québec dans les services dirigés aux demandeurs d'asile. Ce resserrement des services auprès des demandeurs d'asile est corroboré par certains informateurs gouvernementaux rencontrés. Un représentant municipal en donne cette explication :

À l'époque, quand le bureau [d'aide sociale pour les réfugiés] a été créé, ils avaient accès aux mesures d'employabilité. Ils avaient donc accès à des mesures de francisation. On faisait de la francisation et même certains qui avaient des permis de travail avaient accès à des programmes d'aide à l'intégration à l'emploi. On a donc essayé le plus possible d'aider cette clientèle-là à intégrer le marché du travail et à se franciser et donc, à participer à la vie économique, sociale et culturelle. Ça a fonctionné jusqu'en 1996, je crois, et le gouvernement a ensuite revu la réglementation et a décidé que les demandeurs d'asile n'étaient plus admissibles aux mesures actives d'employabilité. Les demandeurs d'asile n'avaient donc plus le droit de participer aux stages en milieu de travail, ni à la francisation sauf à celle à temps partiel. À partir de là, ça a été plus difficile parce que le gouvernement ne voulait plus investir en faveur d'une clientèle sans savoir si celle-ci allait avoir le statut et continuer à rester au Canada ou si elle risquait d'être expulsée. La Ville a fait des pressions, je pense, sur le gouvernement du Québec pour assouplir la loi, pour les rendre admissibles à certaines mesures, mais ça a toujours été un problème.

Un représentant fédéral constate aussi un manque évident de volonté du gouvernement du Canada d'investir dans l'intégration des demandeurs d'asile avant leur acceptation comme personne protégée ${ }^{29}$ au Canada :

The language programs are not just for refugees, those are for immigrants as part of the integration package. But, for asylum seekers, the assumption is that they are not, they have no status in Canada in terms of needing to be integrated until a determination is made on their claim. And so, until they go before the Immigration [and Refugee] Board of Canada (IRB) ${ }^{30}$ and a determination is made, the only benefit that they have [from the federal government] is the Emergency and Essential Healthcare through the IFH program.[...] See, the problem with asylum seekers is the obligation to integrate, and until there is some kind of a definition for obligation to integrate, they are just what we say they are - asylum seekers. And if you look at the statistics, $60 \%$ of those people seeking asylum are failed, they don't actually pass the IRB. So, it is very hard to justify spending gobs of money setting up special programs for a population for whom $60 \%$ didn't qualify in the first place. So that is where it becomes very hard. It is much easier for us to focus on the people for whom Canada has already said, "Yes, you meet this requirement and we will allow you to stay in Canada, and we will take responsibility for your integration." Until they have reached that decision, it becomes very difficult.

Aux dires des informateurs-clés, les difficultés d'accès aux services font en sorte qu'elles nuisent au processus d'incorporation du demandeur d'asile, comme l'explique un représentant municipal :

C'est sûr que ça retarde le processus d'intégration dans la mesure où ces gens-là n'ont pas accès à des mesures comme des stages en milieu de travail. Une personne qui serait revendicatrice [du statut de réfugié] ne pourrait pas avoir accès, car pour les stages, il y a une entente avec Emploi Québec pour le financement du salaire, qui est défrayée à travers le programme de subvention salariale et celui-ci est pour des clientèles « immigrants reçus », « citoyens canadiens ». Un revendicateur n'a pas accès à ce programme, donc il ne pourrait pas faire un stage. C'est donc clair que ça retarde son intégration au point de vue économique. 
Cependant, à long terme, ce retard dans le processus d'incorporation du demandeur d'asile, une fois que celui-ci est reconnu comme réfugié au Canada, aura inévitablement des répercussions sur la société québécoise. L'intervenant d'un groupe de défense des droits des réfugiés explique :

Le fait qu'ils n'ont pas le droit de prendre les cours de langue ou bien les cours d'informatique ou d'autres choses, ça leur donne l'idée que "Vous ne le méritez pas ». Ça lance l'idée que peutêtre «Quand vous êtes réfugié, on va vous accueillir, mais entre temps, vous êtes un demandeur et on n'a pas besoin de vous ». Ça, je crois que c'est couper le nez de société, je crois que c'est stupide en fin de compte. Si une personne ne peut pas parler pour demander un billet de transport ou pour demander de la nourriture, c'est stupide. Pour les enfants qui commencent à l'école, pour leurs parents qui ne peuvent pas leur parler ou un mot du professeur, ça n'a pas de bon sens. Mais c'est juste un manque de ressources financières et ça répond aux besoins des revendicateurs, revendicatrices, alors pourquoi pas ? Surtout comme j'ai dit, c'est pas une question de $10 \%$ qui vont être acceptés et $90 \%$ expulsés, pas du tout. C'est loin de ça. Alors cet aspect de prévention des problèmes à long terme, je pense à long terme, ça va nous donner : ils vont travailler plus vite, moins de problèmes à la maison peut-être. Ils vont payer les impôts, ils vont partager, s'impliquer dans la société. Et pour nous tous, l'apprentissage de langue ça va plus vite quand tu travailles. Alors, c'est évident qu'un grand nombre de personnes qui rentrent vont rester. Et si on ne dépense pas trop au début, on va avoir des problèmes à long terme. C'est un peu préventif, c'est de façon parallèle : Est-ce que vous voulez guérir une maladie ou est-ce que vous voulez prévenir une maladie?

Les difficultés d'accès aux services se santé sont soulignées par les informateurs clés comme l'un des gros problèmes. Le Programme fédéral de santé intérimaire (PFSI) administré par Citoyenneté et Immigration Canada (CIC) garantit que les services médicaux essentiels et les services d'urgence sont offerts aux demandeurs d'asile qui se trouvent au Canada et qui ne sont pas encore couverts par le régime provincial de soins de santé ${ }^{31}$. Toutefois, plusieurs intervenants ont été confrontés dans leur pratique à un refus de leur clientèle aux soins de santé en raison du manque d'acceptation du PFSI dans plusieurs réseaux de la santé. Comme l'explique l'intervenant d'un abri pour les réfugiés :

C'est vraiment le manque d'accès, que ce soit aux services de santé, c'est tellement difficile de faire accepter, surtout ici dans le quartier. La plupart des cliniques ne vont pas accepter le PFSI, le programme fédéral de santé. Ils ne veulent pas l'accepter. [... ] Mais il faut qu'ils reconnaissent ce papier-là qu'on leur donne, parce que quand ils arrivent ici, c'est le seul papier qu'ils ont. Ils n'ont pas accès à rien d'autre que le PFSI, donc c'est important que les hôpitaux, les cliniques, tout ça, reconnaissent ça parce que les gens n'ont vraiment, vraiment pas autre chose.

D'ailleurs, selon les dires des répondants, le manque d'accessibilité aux services de santé est d'autant plus pénible pour les demandeurs d'asile lors de leur arrivée au Québec, notamment en raison des problèmes de santé mentale liés aux traumatismes et à la violence subis dans leur pays d'origine.

\section{Le long délai de traitement de la demande du statut de réfugié et la séparation familiale}

Les intervenants communautaires consultés ont déclaré à l'unanimité que le long délai de traitement de la demande du statut de réfugié est un obstacle difficile à surmonter pour les demandeurs d'asile au Canada. En effet, le délai moyen de traitement de la demande à la CISR pour l'exercice 2005-2006 est de 12,5 mois (en baisse par rapport aux 13,6 mois pendant l'exercice 2004-2005 et 14,2 en 2003-2004) ${ }^{32}$. Bien que plusieurs informateurs-clés aient constaté comme un élément positif la diminution du délai moyen de traitement ${ }^{33}$, pour tous les cas, cette attente et cette incertitude génèrent de l'anxiété chez le demandeur. L'intervenant d'un service d'intégration des nouveaux arrivants décrit cette période de la façon suivante :

J'ai reçu des revendicateurs l'aveu que l'attente avait été assez longue. Ils ont passé à travers beaucoup de choses, beaucoup de peine. Il y a même eu des dépressions. Je les entends dire "Quand est ce que je vais être accepté ? ", " Il faut que j’aie la réponse que je suis accepté ", "Qu'est-ce qui va m’arriver ? ". Alors, je sens qu'il y a une tension par rapport à ça. Ça fait quelques personnes que j'ai rencontrées pour qui l'attente a été assez difficile.

L'intervenant ajoute que cette anxiété vécue par les demandeurs d'asile peut aussi avoir des effets négatifs sur le processus d'incorporation de leurs enfants :

J'ai connu des jeunes âgés de 6 à 12 ans. Ces jeunes vont à l'école et puis ils ont des difficultés. Ils comprennent le stress que vivent leurs parents, ceux qui arrivent, bien sûr, avec des parents, alors il y a des difficultés d'apprentissage.

Cette période d'attente est d'autant plus difficile pour le demandeur lorsque sa famille est encore dans son pays d'origine, car cela implique souvent une longue séparation avec leur conjoint et leurs enfants. Certains intervenants témoignent de la difficulté pour les réfugiés, une fois accep- 
tés au Canada, de faire venir leur famille, comme le décrit l'intervenant d'un service d'intégration des réfugiés et immigrants :

Les revendicateurs fuient leur pays d'où ils sont et dans bien des cas, se sont des personnes qui arrivent, pas nécessairement avec leur famille et donc c'est comme, beaucoup de stress pour eux. Ils sont ici, les autres membres de la famille sont au pays. Et ici, tant que ces revendicateurs n'ont pas le droit de travailler, parce qu'ils n'ont pas de travail à cause que la procédure traîne au niveau de l'immigration avant... qu'ils puissent passer à la CISR pour voir s'ils sont admissibles, s'ils sont vraiment réfugiés ou pas. Alors c'est un processus qui est quand même très long. Moi j'ai des cas où les personnes ont attendu deux ans avant de passer à la CISR ... pour pouvoir être accepté. Une fois que c'est fait, là il faut voir maintenant. «J'ai ma famille là-bas, il faut que ma famille me rejoigne ici, c'est difficile. » D'abord, ils n'ont pratiquement rien pour les frais pour l'étude de dossier pour la demande de résidence permanente. «Comment vais-je payer la demande de résidence permanente? ». Et il y a des fois où il $\mathrm{y}$ en a qui vont emprunter de l'argent. Des fois il y en a qui arrivent à travailler. C'est pas suffisant pour leurs propres besoins ici et aussi pour subvenir aux besoins des membres de la famille au pays, alors c'est compliqué,c'est difficile. Je reçois des appels et des appels, "Qu'est-ce que je fais, comment je vais faire? ", «Il $\mathrm{y}$ a plein de problèmes qui se passent dans mon pays et je sais pas où est ma famille ». C'est vraiment très, très, très pénible et stressant, tant pour eux autres que pour nous autres comme intervenantes. Alors, ce n'est pas normal qu'on les fasse attendre pendant tout ce temps. Il doit y avoir une façon, un moyen d'accélérer ce processus en tenant compte des membres de la famille. Soit que si ce n'est pas possible de faire plus vite, mais d'au moins donner la chance aux revendicateurs, pour qu'ils puissent faire venir les autres membres de la famille.

Les témoignages révèlent que les conséquences de la séparation familiale ${ }^{34}$ sont multiples pour les demandeurs qui revendiquent le statut de réfugié et qui se trouvent dans une telle situation. Les retards dans la réunification des familles ${ }^{35}$ peuvent d'ailleurs avoir un impact important dans le processus d'incorporation du réfugié et de sa famille une fois arrivés au Québec ${ }^{36}$. Voici comment l'intervenant d'un abri pour les réfugiés décrit les impacts de cette longue séparation familiale :

C'est difficile parce que souvent, le temps qu'elles attendent leur audience, elles n'ont même pas les moyens d'accumuler de l'argent pour pouvoir payer pour leur résidence quand ce sera le temps, donc ça créé beaucoup de problèmes après. C'est vraiment pas évident, juste l'attente, attendre que ce soit, que ce soit n'importe quoi, faut toujours attendre, mais surtout pour l'audience, pour la réponse, la décision qui a été prise à l'audience. C'est tellement long. C'est hallucinant, c'est fou comment c'est long. Puis attendre d'être réunifié avec leur famille aussi après. Parce que eux souvent ils se disent « Bon, d'ici deux ans ma famille va être là ", mais des fois ça peut prendre jusqu'à six ans avant que tu revoies tes enfants et ça, ça crée d'autres problèmes, parce que quand les enfants arrivent, les familles éclatent.

L'intervenant du service d'intégration des nouveaux arrivants confirme les effets néfastes de la séparation des familles une fois que celles-ci arrivent au Canada :

La réunification familiale, ça c'est très pénible parce que les gens là-bas ils ne savent pas. Des fois ils disent « Mon mari ne veut plus que je le rejoigne » et c'est la même chose pour les enfants, bien que ce n'est pas le cas. Alors donc ces personnes, quand elles arrivent, ce qui arrive ici c'est que les enfants ne reconnaissent plus le père ou la mère, un des deux qui arrive avant. Parce que, après deux ans, trois ans, « c'est plus mon père, c'est plus ma mère ». Alors ils n'écoutent pas et donc là c'est une autre chose qui est soulevée. Et c'est une grosse complication pour que le père ou la mère puisse arriver à faire que l'enfant comprenne. Même dans le couple il y a des choses qui ne marchent pas et ce qui arrive c'est qu'il y a des cas de séparation. Ils se séparent de la famille alors c'est tout à recommencer. C'est vraiment encore compliqué et chacun fait son chemin.

Au sujet du délai d'attente, plusieurs intervenants dénoncent une situation encore plus dramatique pour les ressortissants de pays moratoires. Les personnes qui sont sous moratoire ${ }^{37}$ sont des personnes qui se sont vu refuser le statut de réfugié. Cependant, le gouvernement canadien a reconnu qu'on ne devrait pas les renvoyer vers leurs pays ${ }^{38}$ à cause de la situation de risque généralisé auquel est exposé l'ensemble de la population civile. Ces personnes peuvent rester au Canada pendant une période de temps qui peut aller au-delà de six ans sans avoir un statut particulier sur le territoire.

En conclusion, ce statut limite non seulement l'accès aux services publics et l'incorporation à la société civile, mais aussi la possibilité d'initier les démarches pour une réunification familiale exacerbée par un long délai d'attente. À ceci s'ajoutent les effets néfastes sur la santé et le bien-être des familles.

\section{Le rôle des organismes communautaires dans le processus d'incorporation des demandeurs d'asile}

Les intervenants ont décrit le mandat et les services offerts par leurs organismes, ainsi que les lacunes et les obstacles constatés pour venir en aide aux demandeurs d'asile. La 
plupart des organismes ont comme premier mandat la prestation de services aux nouveaux arrivants, ce qui comprend les résidents permanents et les réfugiés sélectionnés par le Canada à l'extérieur du pays ou les réfugiés acceptés sur place. Cependant, bien que les demandeurs d'asile soient généralement exclus de leurs mandats ou missions, en raison de leur précarité et leurs besoins réels, tous les répondants des organismes rencontrés aident régulièrement les demandeurs d'asile qui sollicitent leur aide, comme l'explique un des intervenants :

Je ne trouve pas ça correct qu'on nous dise de donner seulement le service de logement aux revendicateurs. Ils sont comme tous les immigrants, ils ont besoin de toutes sortes de choses. Si on les regarde bien, ils ont besoin même de plus de choses, en commençant par le support moral, parce que ce sont des personnes qui fuient leur pays donc leurs problèmes sont plus graves que ceux qui arrivent comme indépendants parce qu'ils choisissent de venir et ils sont préparés pour faire le deuil de l'immigration. Tandis que les revendicateurs c'est complètement différent, ils fuient leur pays d'où ils sont, donc il faut qu'on les soutienne, qu'on aide ces personnes et dans bien des cas, ce sont des personnes qui arrivent pas nécessairement avec leur famille et donc c'est comme, beaucoup de stress pour elles. [...] On rencontre aussi des personnes qui ne parlent pas le français. Ici on a plusieurs niveaux de français, on a à peu près une vingtaine de cours par semaine et normalement ces cours sont adressés aux personnes qui ont un statut, pas de revendicateurs. Et quand les revendicateurs sont acceptés comme réfugiés, ils peuvent participer, ils ont droit comme tous les autres immigrants. Mais les revendicateurs, ils ont un peu moins de chance et il arrive quand même que le coordonnateur prenne l'initiative d'accepter quelques revendicateurs.

Dans le but de pallier les restrictions structurelles auxquelles font face les demandeurs d'asile, notamment à cause d'un manque de financement gouvernemental et d'un choix de politique étatique, certains organismes communautaires aident bénévolement les individus dans les processus de détermination du statut de réfugié et d'incorporation à la société québécoise. Ces organismes offrent des services tels que le support moral, l'information et les références, le programme de jumelage social avec des Québécois ou Canadiens, le suivi psychologique, la préparation du dossier de demande du statut de réfugié, l'accompagnement aux audiences devant la CISR. Certains groupes communautaires reçoivent une aide financière afin d'être en mesure d'offrir l'hébergement temporaire et l'aide à la recherche d'un logement.

Toutefois, plusieurs intervenants témoignent leur difficulté à venir en aide à cette clientèle en raison de leurs restrictions budgétaires, de la surcharge de travail et du manque de personnel. De ce fait et pour remédier à ces lacunes, les intervenants ont signalé que les organismes collaborent souvent entre eux afin de pouvoir combler ces insuffisances. En effet, la concertation communautaire en vue d'aider les demandeurs d'asile à intégrer la société québécoise est une pratique courante.

\section{Conclusion}

Cet article a permis d'examiner en partie le processus d'incorporation des demandeurs d'asile au Québec et, dans la mesure où ce processus peut $y$ trouver une application plus générale, dans le reste du Canada. Celui-ci diffère du processus d'intégration en ce qu'il considère le demandeur d'asile comme un citoyen à part entière, c'est-à-dire un membre actif dans la société, ayant des droits et des obligations dès son arrivée et, a priori, non soumis à une discrimination au sein d'une population diversifiée.

Comme les informateurs-clés en témoignent, les demandeurs d'asile sont confrontés à différentes barrières lors du processus d'incorporation. La réticence du gouvernement à investir auprès de cette population autant qu'il le fait pour les autres catégories d'immigrants s'explique par le risque de la voir quitter le pays lors des expulsions ou des déportations, du fait que le taux d'acceptation des demandes de statut de réfugié frôle le $50 \%$. Le processus qui conduit à une décision gouvernementale cause aussi de nombreux préjudices à la population acceptée puisque les demandeurs d'asile en attente d'une décision vivent une situation d'altérité et de marginalisation sociale, quelle que soit la province d'accueil. D'autre part, ceux qui seront acceptés seront retardés dans leur processus d'incorporation ce qui produira un impact négatif sur le tissu culturel et socioéconomique, tant régional que national. Il est aussi important de mentionner que ce long délai de traitement de la demande du statut de réfugié, l'incertitude entourant la décision d'acceptation et la séparation familiale aggravent les difficultés que vivent les demandeurs d'asile.

Le rôle des organismes communautaires dans le processus d'incorporation des demandeurs d'asile est très important, car leurs services pallient les lacunes des politiques gouvernementales et municipales. Ces organismes ont un manque flagrant et chronique de financement et de personnel. Une concertation intersectorielle, une collaboration et une communication de meilleure qualité entre les divers pouvoirs publics et les organismes sont aussi requises et mentionnées systématiquement dans les récits des intervenants.

Les constats des chercheurs montrent que les limitations appliquées aux demandeurs d'asile définissent leur situation et minimisent leurs contributions. L'ostracisme 
déterminé par leur statut provisoire et caractérisé par une absence d'action et une exclusion participative dans la société d'accueil devient démobilisateur. Le processus d'incorporation favorise l'examen des rapports qui sous-tendent les mécanismes structurels, politiques et sociaux définissant les politiques d'intégration. Ce dispositif permet d'élaborer les stratégies nécessaires pour pallier les politiques administratives insuffisantes et diminuer l'exclusion des individus déjà soumis à la souffrance dans leur pays d'origine et au déracinement dans la société d'accueil.

L'examen du mode d'incorporation montre que les demandeurs d'asile sont particulièrement conditionnés par les instances qui élaborent et gèrent les politiques et les lois. Ces rapports influencent intimement leur destinée en limitant leur niveau de participation et jouent sensiblement sur leur capital humain et symbolique. La distribution irrégulière des droits tant symboliques que pratiques s'oppose à la réalité où leur présence ne peut être niée en leur déférant un statut moindre. D’autre part, les politiques gouvernementales interviennent de façon fondamentale dans le processus migratoire en raison des structures qu'elles mettent en place. Si le processus d'incorporation est bien un processus multidimensionnel, il est possible d'envisager le degré de participation, voire d'appartenance des demandeurs d'asile dans la société d'accueil. L'hiatus entre l'application des politiques, la pratique et les besoins de cette population particulièrement sensible dessert les objectifs du pays d'accueil qui sont, au préalable, de protéger et de traiter tous ses résidents d'une façon impartiale. En outre, elle amenuise les capacités des demandeurs d'asile par cette étanchéité.

Le processus d'incorporation permet d'approfondir le niveau de responsabilité du pays d'accueil engagé dans le respect des droits accordés aux résidents qui demeurent sur son territoire. Le pays d'accueil doit garantir cette protection et l'accès aux ressources selon l'élargissement des droits et des discours transnationaux. Cette garantie s'étend implicitement aux demandeurs d'asile malgré la forme provisoire de leur statut. Les initiatives prises par les politiques et les expériences des demandeurs d'asile s'inscrivent dans le répertoire institutionnel du système des politiques d'accueil. Cet appareil concerne le modèle politique, les structures organisationnelles et les discours sur l'appartenance qui sont reconfigurés selon les prescriptions modernes de l'élément global du développement de la notion universelle de la personne influençant les politiques nationales ${ }^{39}$.

Pour les demandeurs d'asile, ce processus d'incorporation commence dès la réception dans le pays d'accueil et s'achève dans un premier temps lorsqu'ils sont acceptés comme réfugiés. Pendant ce laps de temps qui peut durer des années, cette population appartient de facto au système politique et participe à la société d'accueil. La méthode de réception inscrite dans les politiques migratoires et l'expérience éprouvée par les demandeurs d'asile interfèrent dans la rencontre avec le groupe dominant, d'où la nécessité d'élever l'analyse à une unité sociale plus large qui comprend l'État et la société civile. Ainsi, le concept d'incorporation permet d'apprécier tous les processus par lesquels les nouveaux arrivants deviennent des membres à part entière de la société d'accueil dans une vision plus globale. Cette perspective permet de prendre en compte les dynamiques politiques enchevêtrées dans l'activité sociale où interagissent les demandeurs d'asile et les membres de la société d'accueil en préconisant un accès commun aux droits et privilèges puisqu'ils deviennent des acteurs sociaux dès leur arrivée ${ }^{40}$. Cette conception évite de catégoriser le demandeur d'asile dans une altérité qui le classe dès le départ dans une situation inférieure en prenant acte de sa participation et de sa perception d'acceptation par les membres de la société d'accueil. Ainsi, ce procédé s'intéresse à tous les aspects de la vie et aux rapports structurels dans lesquels sont engagés les individus dans une société civile et politique $^{41}$. Le processus d'incorporation, dès la mise en cheminement de la demande d'asile, encourage davantage la participation active, l'égalité et le dialogue entre les cultures dans une société caractérisée par la diversité ${ }^{42}$.

\section{Notes}

1. Les réfugiés et les personnes en situation semblable sélectionnés à l'étranger, accueillis à la charge de l'État ou dans le cadre du programme de parrainage collectif, sont exclus de cette analyse. Reconnus réfugiés par le gouvernement fédéral avant leur arrivée sur le sol canadien, ces derniers bénéficient dès leur entrée au pays du statut de résident permanent. De ce fait, les réfugiés parrainés au Canada, contrairement à notre groupe d'étude, bénéficient de l'aide à l'établissement dès leur arrivée au Canada, bien que certains d'entre eux puissent ignorer leurs droits.

2. Convention relative au statut des réfugiés, adoptée le 28 juillet 1951 par une conférence de plénipotentiaires sur le statut des réfugiés et des apatrides convoquée par l'Organisation des Nations Unies en application de la résolution 429 (V) de l'Assemblée générale en date du 14 décembre 1950. Convention relative au statut des réfugiés, 28 juillet 1951, 189 U.N.T.S. 150, Can. T.S. 1969/6 (Entrée en vigueur le 22 avril 1954; accession par le Canada le 2 septembre 1969).

3. Personne visée par la Convention contre la torture et autres peines ou traitements cruels, inhumains ou dégradants, signée à New York le 10 décembre 1984 et entrée en vigueur le 26 juin 1987.

4. Toute personne peut présenter une demande en vue de rester au Canada pour des motifs d'ordre humanitaire. Ceci comprend les personnes qui demandent l'asile, mais dont la de- 
mande n'a pas été approuvée par la Commission de l'immigration et du statut de réfugié. Les demandes de résidence permanente pour des motifs d'ordre humanitaire ne sont approuvées que dans des circonstances exceptionnelles. Il peut arriver que le traitement d'une demande prenne plusieurs années et il n'existe pas de droit d'appel d'une décision négative de Citoyenneté et Immigration Canada. Dans certains cas, toutefois, il existe la possibilité de demander à la Cour fédérale du Canada de contrôler la décision. Citoyenneté et Immigration Canada (CIC), Examen des considérations humanitaires, $<$ http://www.cic.gc.ca/FRANCAIS/ausujet/immigration/hu manitaires.asp> (accédé le 18 septembre 2007).

5. De nombreuses personnes issues de pays visés par un moratoire ont dû mettre leur vie en suspens plusieurs années depuis qu'elles sont au Canada. Elles se trouvent dans un vide juridique parce qu'elles n'ont pas obtenu le statut de résident permanent; pourtant, elles ne peuvent retourner dans leur pays en raison de l'insécurité qui y règne, un danger d'ailleurs reconnu par le gouvernement du Canada, qui a imposé un moratoire sur les renvois dans ces pays. (CCR, 2006). Des vies en suspens. Les limites des considérations humanitaires, 6 septembre 2006, Conseil canadien pour les réfugiés (CCR), $<$ http:// www.ccrweb.ca/communviessept06.html> (accédéle 3 octobre 2007).

6. Consulter les accords conclus avec les provinces et les territoires: Accord Canada-Québec relatif à l'immigration et à l'admission temporaire des aubains (signé le 5 février 1991); Accord Canada-Manitoba en matière d'immigration (signé le 22 octobre 1996 et renouveléle 6 juin 2003); Accord Canada-Saskatchewan sur l'immigration (signé le 16 mars 1998 et renouvelé le 7 mai 2005); Accord relatif à la collaboration entre le Canada et la Colombie-Britannique en matière d'immigration (signé le 19 mai 1998 et modifié le 15 juin 2005); Entente Canada-Nouveau-Brunswick sur les candidats de la province (signé le 22 février 1999 et modifié le 29 mars 2005); Entente CanadaTerre-Neuve et Labrador sur les candidats de la province (Signé le $1^{\text {er }}$ septembre 1999. Lettre de prolongation : 10 janvier 2005); Accord relatif à la collaboration entre le Canada et l'îledu-Prince-Édouard en matière d'immigration (signé le 29 mars 2001); Accord de collaboration Canada-Yukon en matière d'immigration (signé le 2 avril 2001); Entente Canada-Alberta sur les candidats de la province (signé le 2 mars 2002. Lettre de prolongation : 11 avril 2005); Entente Canada-Nouvelle-Écosse sur les candidats de la province (signé le 27 août 2002); Accord Canada-Nouvelle-Écosse sur l'immigration (signé le 19 septembre 2007).

7. Accord Canada-Québec relatif à l'immigration et à l'admission temporaire des aubains, signé le 5 février 1991.

8. Loi sur l'immigration au Québec, L.R.Q., chapitre I-0.2.

9. Marie Lacroix, "L'expérience des demandeurs d'asile : vers l'élaboration de nouvelles pratiques sociales," Nouvelles pratiques sociales $16, \mathrm{n}^{\circ} 2$ (2003) : 188.

10. Jacqueline Oxman-Martinez et Jill Hanley, "L’identité assignée du statut d'immigration précaire et l'accès aux services de santé : la construction sociale de l'exclusion," dans Éthique de l'altérité, F. Saillant (dir.) (Québec: Presses de l’Université Laval, sous presse).

11. Par exemple, les soins en matière de contraception, de prénatalité et d'obstétrique, les médicaments essentiels sur ordonnance, etc.

12. Jacqueline Oxman-Martinez, Jill Hanley, Lucyna Lach, Nazilla Khanlou, Swarna Weerasinghe et Vijay Agnew, "Intersection of Canadian policy parameters affecting women with precarious immigration status: A baseline for understanding barriers to health," Journal of Immigrant Health 7, $\mathrm{n}^{\circ} 4$ (2005) : 247-58.

13. Commission de l'immigration et du statut de réfugié du Canada (CISR), Statistiques 2006 pour la Commission de l'immigration et du statut de réfugié, Décisions de la Section de la protection des réfugiés, <http://www.irb-cisr.gc.ca/fr/index_f.htm> (accédé le 13 mars, 2007).

14. Elizabeth Mestheneos et Elizabeth Ioannidi, "Obstacles to refugee integration in the European Union member states," Journal of Refugee Studies 15, no 3 (2002) : 304-20.

15. Stephen Castles, Maja Korac, Ellie Vasta et Steven Vertovec, Integration: Mapping the Field, avec l'aide de Katrin Hansing, Fiona Moore, Emma Newcombe, Lucy Rix et Soojin Yu, rapport d'un projet réalisé par le Centre for Migration and Policy Research et le Refugee Studies Centre de l'University of Oxford pour le Immigration Research and Statistics Service (IRSS) du Home Office, décembre 2002, Home Office Online Report 28/03, <http://www.homeoffice.gov.uk/rds/pdfs2/rdsolr2803. doc $>$ (accédé le 16 mars 2007).

16. Oxman-Martinez et Hanley, "L'identité assignée du statut d'immigration précaire."

17. Jacqueline Oxman-Martinez et Jill Hanley, "Health and Social Services for Canada's Multicultural Population: Challenges for Equity." Policy Forum. Canada 2017. Serving Canada's multicultural population for the future. Policy Forum. Discussions Papers. Canadian Heritage, Multiculturalism. March 22-23 2005: 19-32, <http://www.pch.gc.ca/progs/multi/ canada2017/index_e.cfm > (accédé le 3 octobre 2007).

18. Mestheneos et Ioannidi, 304-20; Lacroix, M., "Social work with asylum seekers in Canada : The case for social justice," International Social Work 49, no 1 (2006) : 19-28; OxmanMartinez et Hanley, supra note 10.

19. Mestheneos et Ioannidi, ibid.; Lacroix, ibid.

20. Par exemple, un manque de financement public.

21. Lacroix, "L'expérience des demandeurs d'asile," 188; Lacroix, "Social work with asylum seekers in Canada," 19-28.

22. Charte canadienne des droits et libertés, édictée comme l'annexe B de la Loi de 1982 sur le Canada, 1982, ch. 11 (R.-U.), entrée en vigueur le 17 avril 1982; Singh c. Ministre de l'Emploi et de l'Immigration, [1985] 1 R.C.S. 177.

23. Yasemin Nuhoglu Soysal, Limits of Citizenship: Migrants and Postnational Membership in Europe (Chicago et Londres : University of Chicago Press, 1994).

24. Accord Canada-Québec, supra note 7.

25. Mestheneos et Ioannidi, 304-20; Lacroix, "Social work with asylum seekers in Canada," 19-28; Oxman-Martinez et Hanley, "L’identité assignée du statut d'immigration précaire." 
26. Oxman-Martinez et Hanley, supra note 17; Oxman-Martinez et Hanley, supra note 10; Lacroix, supra note 9; Lacroix, supra note 18.

27. Barney Glaser et Anselm Strauss, The Discovery of Grounded Theory (Chicago : Aldine Publishing Company, 1967); A. Michael Huberman et Matthew B. Miles, "Data management and analysis methods," Handbook of Qualitative Research, Norman K. Denzin et Yvonna S. Lincoln (dir.), (Londres : Sage, 1994), 428-44.

28. Le Programme canadien de réinstallation des réfugiés et des personnes protégées à titre humanitaire comprend l'octroi des fonds en vue de contribuer au paiement des frais liés à l'accueil du réfugié à l'aéroport ou au point d'entrée; l'hébergement temporaire; l'aide nécessaire pour trouver un logement permanent; l'achat des articles de base pour la maison et la prestation d'une orientation générale de la vie au Canada. Les fonds octroyés sont aussi utilisés pour fournir au réfugié un soutien de revenu pour une période pouvant atteindre un an, ou jusqu'à ce que la personne puisse subvenir seule à ses besoins, selon ce qui se produit en premier.

29. Loi sur l'immigration et la protection des réfugiés, L.C. (2000), ch. 27 [LIPR], article 95 (2): « est appelée personne protégée la personne à qui l'asile est conféré ».

30. Commission de l'immigration et du statut de réfugié au Canada (CISR), <http://www.irb-cisr.gc.ca/en/index_e.htm $>$ (accédé le 26 septembre 2007).

31. Citoyenneté et Immigration Canada, Droit à l'emploi, à l'éducation et aux services de santé, <http://www.cic.gc.ca/francais/ refugies/asile-5.html> (accédé le 31 mars 2007).

32. Lacroix, "L'expérience des demandeurs d'asile," 188.

33. La Section compte atteindre un délai moyen de traitement de 11 mois pour l'exercice 2006-2007 pour en arriver un jour à un délai moyen de traitement de six mois.

34. Consulter le site du Conseil canadien pour les réfugiés, « $T u$ me manques... ", Campagne en faveur de la réunification familiale, <http://www.web.net/ ccr/reunificationfr.html> (accédé le 3 octobre 2007).

35. D'ailleurs, en matière d'immigration, la LIPR a pour objet de veiller à la réunification des familles au Canada (Art. 3. (1) d)).

36. L'importance de la réunification familiale dans le cadre de l'intégration est reconnue dans les objectifs de la $L I P R$, qui stipule que la loi vise à « encourager l'autonomie et le bien-être socioéconomique des réfugies en facilitant la réunification de leurs familles au Canada » (art. 3(2)(f)), tandis que la Loi sur l'immigration au Québec, en vertu de l'article 3 alinéa b), a pour objet de faciliter la réunion au Québec des citoyens canadiens et résidents permanents avec leurs proches parents de l'étranger.

37. Règlement sur l'immigration et la protection des réfugiés, DORS/2002-227, art. 230.

38. Actuellement (mars 2007), en raison du risque généralisé qui y prévaut, le gouvernement du Canada impose un moratoire sur les renvois vers huit pays : l'Afghanistan, le Burundi, Haïti, l'Irak, le Libéria, la République démocratique du Congo, le Rwanda et le Zimbabwe. Source : Conseil canadien pour les réfugiés, campagne Des vies en suspens, $<$ http://www.ccrweb. ca/fra/accueil/accueil.htm> (accédé le 17 avril 2007).

39. Mestheneos et Ioannidi, 304-20; Lacroix, "Social work with asylum seekers in Canada," 19-28; Oxman-Martinez et Hanley, "L’identité assignée du statut d'immigration précaire."

40. Supra note 11 .

41. Supra note 7.

42. Oxman-Martinez, Hanley, Lach, Khanlou, Weerasinghe et Agnew, 247-58.

Jacqueline Oxman-Martinez, Ph.D. (sociologie, Université de Montréal), est professeure associée et chercheure invitée au Centre de recherche interdisciplinaire sur la violence familiale et la violence faite aux femmes (CRI-VIFF) Université de Montréal. Ses recherches portent sur la traite des femmes et des enfants et les problématiques reliées au statut précaire d'immigration.

Estibaliz Jimenez, Ph.D. (criminologie, Université de Montréal), enseigne le droit de l'immigration et des réfugiés à la Faculté de Droit de l'Université de Sherbrooke et elle travaille comme agente de recherche avec J. Oxman-Martinez au CRIVIFF, Université de Montréal

Jill Hanley, Ph.D. (travail social, Université de Montréal), est professeure adjointe à l'École de Travail Social de l'Université McGill. Ses recherches portent sur les questions de migration, en particulier la migration irrégulière, et ses liens avec les politiques d'immigration et d'accès aux droits sociaux. L'action collective pour la défense des droits des migrants est un de ses intérêts particuliers et elle s'engage également auprès de groupes communautaires travaillant en ce sens.

Isabelle Bohard est étudiante à la maîtrise en anthropologie à l'Université de Montréal. Elle travaille comme auxiliaire de recherche avec J. Oxman-Martinez au CRI-VIFF.

(C) Jacqueline Oxman-Martinez, Estibaliz Jimenez, Jill Hanley and Isabelle Bohard, 2007. This open-access work is licensed under a Creative Commons Attribution-NonCommercial 4.0 International License, which permits use, reproduction and distribution in any medium for noncommercial purposes, provided the original author(s) are credited and the original publication in Refuge: Canada's Journal on Refugees is cited. 